\section{Conocimientos y práctica clínica de los proveedores de salud para la prevención del embarazo adolescente según marco legal chileno}

\author{
INGRID LEAL ${ }^{1, \mathrm{a}}$, CAROLINA LUTTGES ${ }^{1, \mathrm{~b}}$, \\ PAULINA TRONCOSO ${ }^{2}$, CAROLINA LEYTON ${ }^{1, b}$, \\ TEMISTOCLES MOLINA ${ }^{1, \mathrm{c}}$, PAMELA EGUIGUREN ${ }^{3, \mathrm{~d}, \mathrm{e}}$

\section{The legal framework to prevent teenage pregnancies}

Background: There are legal regulations about sexual and reproductive rights of adolescents. However, this legal framework $(L F)$ may have contradictory elements: there are laws assuring confidentiality and access to contraception at any age but there are other laws that consider any sexual contact with an adolescent younger than 14 a sexual assault, whose report to the legal authorities in mandatory. Aim: To explore the knowledge and clinical practice of primary health care (PHC) providers regarding prevention of teenage pregnancy. Material and Methods: Qualitative study collecting data using semi-structured interviews made to midwives and directors of PHC centers. Analysis of the data was based on Grounded Theory. Results: There is a differentiated clinical care for pregnancy prevention among adolescents if they are over 14 years old. This is due to the LF, specifically to the sexual crime's law $(19,927)$ and the law about regulation of the fertility $(20,418)$. The differences affect health care, access and counseling about contraception and confidentiality. Healthcare of teenagers under the age of 14 is perceived as problematic for providers, due to the possible legal implications. Conclusions: The LF causes insecurity on health care providers and derives in a differentiated clinical approach according to the patient's age. This is a barrier to provide timely and confidential access to counseling and contraception.

(Rev Med Chile 2016; 144: 577-584)

Key words: Legislation; Legislation, medical; Pregnancy in Adolescence; Reproductive Rights.

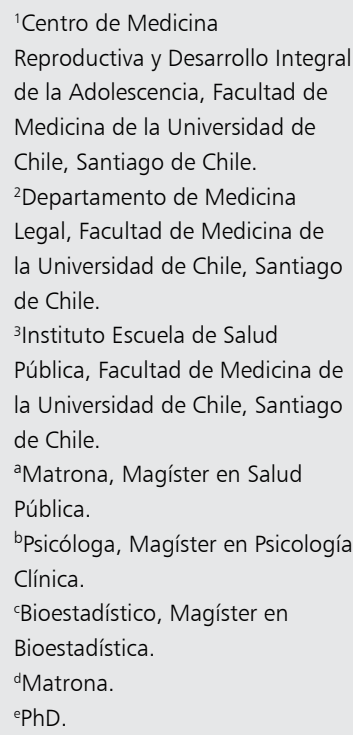

Fuente de financiamiento: el financiamiento se obtuvo en el IX concurso FONIS-CONICYT. Esta institución no influyó en ninguna de las etapas del estudio.

Recibido el 14 de enero de 2015, aceptado el 17 de marzo de 2016.

Correspondencia a: Ingrid Leal Fuentes Av. Profesor Alberto Zañartú 1030,

Independencia, Santiago de Chile.

igleal@med.uchile.cl
E l embarazo adolescente sigue siendo un tema no resuelto que replica las inequidades sociales. Los adolescentes ven disminuidas las posibilidades de ejercer sus derechos en salud porque los servicios de salud no se adecúan a sus necesidades y expectativas ${ }^{1-4}$. El desconocimiento, una comprensión parcial o inadecuada de la normativa regulatoria en salud sexual y reproductiva (SSR) adolescente, de los proveedores de salud y las autoridades locales, pudieran estar actuando como barrera para la atención clínica en la prevención del embarazo, aumentando el riesgo y vulnerabilidad de los adolescentes ${ }^{2,5}$. Un estudio sobre seguimiento de la Ley 20.418, que fija las normas sobre información, orientación y prestaciones en materia de regulación de la fertilidad, concluye que existe diferencia entre el discurso y la práctica de esta normativa, sobre todo por el potencial quiebre de la confidencialidad ${ }^{3}$. Esta situación es más grave en el caso de menores de 14 años, puesto que exis- 
ten normativas que obligan a denunciar al tomar conocimiento que estos adolescentes han tenido actividad sexual, considerándola como violación en todos los casos (Ley 19.927) ${ }^{6,7}$. La edad de consentimiento sexual varía en los países de la región, siendo 14 años en Perú y 13 años en Argentina ${ }^{8,9}$. Además, ante la solicitud de anticoncepción de emergencia (AE) por parte de estos adolescentes, el proveedor, luego de indicar la anticoncepción, debe informar al adulto que la adolescente señale (Ley 20.418 $)^{10}$. Sin embargo, estas mismas leyes, junto a otras normativas y tratados internacionales suscritos por Chile, como la Convención de los Derechos del Niño, hacen referencia a la protección de los derechos de los adolescentes en salud sexual y reproductiva (DSSR) y a la confidencialidad ${ }^{11,12}$. Este panorama puede ser confuso y difícil de interpretar para proveedores en salud, dándole a la atención de adolescentes una connotación médico legal negativa, dejando el acceso a la atención, al criterio u opinión de cada profesional o autoridad local ${ }^{13,14}$. El objetivo de este estudio fue explorar los conocimientos y práctica clínica de los proveedores de atención primaria para la prevención del embarazo adolescente dentro de este marco legal (ML).

\section{Material y Método}

Diseño cualitativo-descriptivo. Muestreo homogéneo intencionado de matronas/es y directoras/es de centros de Atención Primaria de Salud (APS) de salud de la Región Metropolitana. La recolección de datos fue a través de entrevistas semiestructuradas grabadas en audio, en una sesión durante el año 2012, basadas en un guión temático, previamente aplicado y analizado. El análisis se realizó de acuerdo a la Teoría Fun- damentada ${ }^{15}$, contrastando los datos entre los investigadores y con los participantes, a través de la reducción progresiva de los datos, iniciándose con la codificación abierta, de la cual surgieron categorías iniciales, continuando con la codificación axial, donde se organizaron las categorías en un modelo interrelacionado, alrededor de una categoría central o tema más importante, que se vincula con las demás categorías, agrupadas en categorías de contexto, fundamentos, resultados y consecuencias. De este proceso emergió un modelo que tratará de explicar el fenómeno de estudio. Se procuró un registro descriptivo de los y las participantes, que facilitara la transferibilidad de los resultados. Como apoyo para el análisis se utilizó el software Atlas.ti 11. Quedó constancia del proceso de consentimiento informado a través de la firma de éste. (Comité de Ética Facultad de Medicina Universidad de Chile: No 082-2012).

\section{Resultados}

Se entrevistaron 24 participantes (Tabla 1). El modelo que agrupó a la categoría central, atención clínica para la prevención del embarazo adolescente y las categorías de contexto (forman parte del ambiente o situación que enmarcan a la categoría central), categorías de fundamentos (influyentes o causantes y afectan a la categoría central), categorías de resultados (relacionadas con la implementación de acciones que influye a la categoría central) y categorías de consecuencias (resultantes de las acciones e interacciones de las categorías previas), se presentan en la Tabla 2, luego del proceso de análisis (Figura 1).

Dentro del contexto de centros APS del sistema público, se seleccionaron centros especializados en adolescentes (CEA), no especializados, con alguna instancia exclusiva para la atención de adolescen-

Tabla 1. Descripción participantes

\begin{tabular}{|c|c|c|c|c|c|c|}
\hline \multirow{2}{*}{$\begin{array}{l}\text { Edad } \\
\text { (en años) }\end{array}$} & \multicolumn{3}{|c|}{ Matronas/es } & \multicolumn{2}{|c|}{ Directoras } & \multirow[t]{2}{*}{ Total } \\
\hline & SSM Norte & SSM Sur Oriente & CEA* & SSM Norte & SSM Sur Oriente & \\
\hline $25-34$ & 5 & 1 & - & - & - & 6 \\
\hline $35-44$ & 1 & 2 & 1 & 1 & 2 & 7 \\
\hline $45-54$ & 1 & 1 & - & 3 & 2 & 7 \\
\hline 55 y más & 1 & 1 & 1 & 1 & - & 4 \\
\hline
\end{tabular}

*Centro Especializado para Adolescentes. 
Tabla 2. Proceso y modelo de categorización de los datos

\begin{tabular}{|c|c|c|}
\hline Codificación primer nivel (abierta) & Categoría central & $\begin{array}{l}\text { Codificación segundo } \\
\text { nivel (axial) }\end{array}$ \\
\hline $\begin{array}{l}\text { - Tipo de centro } \\
\text { - Equipo de salud } \\
\text { - Capacitación } \\
\text { - Motivación y aspectos biográficos }\end{array}$ & $\begin{array}{l}\text { Atención para la } \\
\text { prevención del embarazo } \\
\text { adolescente }\end{array}$ & $\begin{array}{l}\text { Contexto de la atención } \\
\text { clínica de adolescentes } \\
\text { en SSR }\end{array}$ \\
\hline $\begin{array}{l}\text { - Lineamientos de la atención en SSR para en la adolescencia } \\
\text { - Marco legal relativo a la SSR adolescente } \\
\text { - Percepción de la sexualidad en la adolescencia: } \\
\text { - Embarazo adolescente } \\
\text { - Conducta adolescente } \\
\text { - Anticoncepción adolescente }\end{array}$ & & $\begin{array}{l}\text { Fundamentos de la } \\
\text { atención clínica de } \\
\text { adolescentes en SSR }\end{array}$ \\
\hline $\begin{array}{l}\text { - Atención diferenciada en salud a adolescentes } \\
\text { - Atención diferenciada en menores de } 14 \\
\text { - Denuncia: } \\
\text { o Embarazos menores } 14 \text { años } \\
\text { o Actividad sexual en menor de } 14 \text { años } \\
\text { o Experiencias de denuncias previas } \\
\text { - Quiebre confidencialidad } \\
\text { - Indicación de anticoncepción }\end{array}$ & & $\begin{array}{l}\text { Resultados en la práctica } \\
\text { clínica }\end{array}$ \\
\hline $\begin{array}{l}\text { - Relación profesional/usuario } \\
\text { - Inseguridad en el ejercicio de la profesión } \\
\text { - Conflicto entre ML y rol profesional }\end{array}$ & & Consecuencias \\
\hline
\end{tabular}

tes o "Espacios Amigables" y no especializados, sin servicios específicos de atención. Desde la entrevistas se desprende que en los centros no especializados, la atención de adolescentes era mayoritariamente realizada por matronas/es que manifiestan interés por este grupo, atribuible a aspectos biográficos, que los lleva a facilitar el acceso de adolescentes que concurren en busca de atención, adaptando el proceso de atención, tanto en lo clínico como en lo administrativo. En este sentido, la experiencia del adolescente para resolver su necesidad de atención estará condicionada al profesional que lo atienda.

Una idea reiterada fue la percepción de falta de herramientas como capacitación en los aspectos legales de la atención de adolescentes.

Los fundamentos que enmarcaban la atención de adolescentes para la prevención del embarazo tenía relación con las creencias de los proveedores acerca de la adolescencia y sus conocimientos del ML. Las miradas de los adolescentes como usuarios/as de servicios de SSR, por parte de los proveedores, iban desde ser sujetos de derechos, integrando la sexualidad como una dimensión del desarrollo normal, hasta un enfoque de riesgo, considerando el ejercicio de la sexualidad adolescente como la génesis de resultados adversos, como la deserción escolar y consumo de drogas.

En relación al ML, se identificaron diferencias en el conocimiento y aplicación de las leyes que regulan la práctica clínica para la prevención del embarazo adolescente, siendo la más conocida la ley que tipifica como delito de violación la actividad sexual con o sin consentimiento en menores de 14 años (Ley 19.927). En general, cuando se trata de menores de 14 años que buscan anticoncepción, se les indicaba volver con un adulto, de manera de respaldar su prestación y no realizar la denuncia y, en otros casos, porque consideraban que el adolescente no será capaz de cumplir las indicaciones. A su vez, existía conocimiento respecto a la obligación de denunciar, la que es realizada en el Ministerio Público o Carabineros. No se observó mayor conocimiento respecto a las consecuencias de no denunciar.

Respecto a la Ley 20.418, existía desconocimiento del derecho a confidencialidad y privacidad de la información en la atención para la regulación de la fertilidad. Existía confusión en esta materia y la legalidad en la entrega de anticon- 


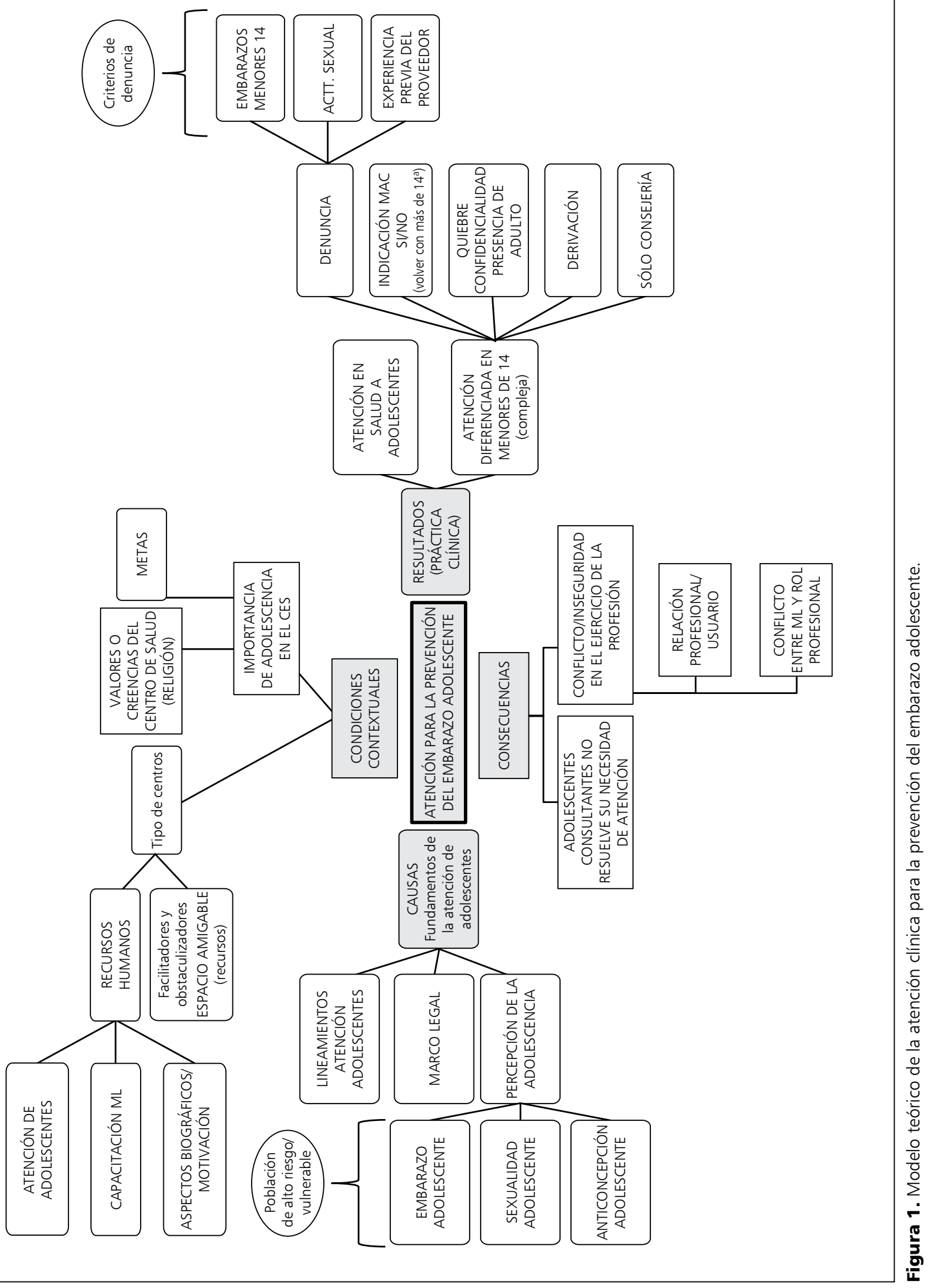


cepción a menores de 14 años, considerando más importante la obligación de denunciar, que mantener la confidencialidad amparada en el secreto profesional, existiendo algunas pocas profesionales que se resguardaban en éste para no realizar la denuncia (Tabla 3). Algunas/os entrevistadas/os hicieron referencia a otras normativas, como la ley de Responsabilidad Penal Juvenil y la ley de Delitos Sexuales, que indican la diferencia de 2 años o más entre adolescentes para ser constitutivo de delito. De esta manera cuando no existe esta diferencia de edad, la entrega de anticoncepción se veía facilitada, para quienes conocían esta normativa. Estos conocimientos generalmente eran transmitidos verbalmente entre las/os profesionales.

Se identificaron diferencias en la atención clínica en el grupo de adolescentes, a partir de las interpretaciones del ML, especialmente sobre abuso sexual, que fija un límite de edad para el consentimiento sexual, distinguiéndose dos grupos, mayores y menores de 14 años. Las diferencias en la atención se relacionan con: atención para la anticoncepción, manejo de la confidencialidad, condicionar la atención a la asistencia de algún adulto y manejo administrativo.

En relación a la indicación de método anticonceptivo (MAC) se identifican diversas estrategias y alternativas: entregar anticonceptivos sin condicionantes; realizar consejería y derivar a otro centro para la indicación de MAC; diferir

\section{Tabla 3. Unidades de significado representativas al $\mathrm{ML}$}

.. hay una parte de la ley, en la que los niños menores de 14 años tienen que ir a juzgado... Cuando tienen actividad sexual"

"Existe libre acceso y derecho a confidencialidad después de los 14 y antes con un adulto"

"Si inicia actividad sexual, nosotros tenemos la obligación de denunciar"

"Entonces la ley dice que si hay 2 años o menos de diferencia, no hay que hacer denuncia, es decir, una niña de 13 años con un niño de 15, o una niña de 13 con un niño de 13, no habría que hacer denuncia"

"Del servicio, del ministerio de salud, ponte tu, el nuevo reglamento de la anticoncepción de emergencia en menores de 14 no ha llegado, eso yo lo baje de internet y lo escuche por la radio, o sea... en base a eso yo... que ni siquiera es un memo, ni algo oficial, por lo tanto yo no tengo mucho donde luchar o ampararme." o condicionar la indicación MAC para cuando se concurra con adulto y postergar la atención para cuando la adolescente tenga más de 14 años. En cuanto a la anticoncepción de emergencia (AE), el proceder era similar. En general, se indicaban MAC fáciles de manejar para la adolescente y que cuiden la privacidad, como inyecciones mensuales. Durante la atención, ya sea a solas o con el adulto acompañante, se entregaba información sobre los aspectos legales. Las prácticas descritas, también fueron identificadas en los CEA.

En cuanto al manejo administrativo, este podía ser modificado, ya sea no registrando la atención o cambiando el motivo de consulta, con el fin de resguardo y evitar involucrase judicialmente. (Tabla 4).

La confidencialidad en adolescentes menores de 14 años no es respetada, a pesar que en general se reconocía que es un factor importante en la relación profesional-usuario. La mayoría de los participantes exigía la presencia de un adulto para la atención clínica. Algunas/os participantes describieron que las adolescentes menores asistían acompañadas por adultos desde un inicio, atribuyendo esta práctica al conocimiento previo por parte de éstas, no teniendo la experiencia de haberlas atendido sin algún adulto.

Fue generalizada la denuncia de los embarazos en menores de 14 años, sin hacer distinciones si la actividad sexual fue consentida o no. En la mayoría de los casos, la matrona le informaba a la adolescente y al adulto que la acompañaba, que debía realizar una denuncia o "notificación" sobre el embarazo, por la normativa legal vigente. El proceso de denuncia era variado, siendo mayoritariamente la matrona quien lo realizaba. Denunciar genera incomodidad, al dar connotación de delito a los embarazos cuando no son producto de una violación, lo que lleva a involucrarse en una instancia judicial. Por otro lado, la mayoría de los profesionales no denunciaban la actividad sexual en menores de 14 años, ya que no la interpretaban desde el ML como delito, o bien no tenían claridad al respecto. Dos entrevistadas realizaban la denuncia de la actividad sexual, exceptuando cuando la diferencia de edad entre la adolescente y la pareja era dos años o menos. Los demás profesionales buscaban diversas formas para no judicializar la atención de estos casos, manteniendo cierto resguardo profesional, como las descritas en la indicación de MAC. 


\section{Tabla 4. Atención diferenciada en menores de 14 años}

\begin{tabular}{|c|c|}
\hline Categoría-Sub categorías & Unidades de significado representativas \\
\hline \multirow{4}{*}{$\begin{array}{l}\text { Denuncia en embarazos } \\
\text { menores o actividad sexual } \\
\text { en menores de } 14 \text { años y } \\
\text { experiencias de denuncias } \\
\text { previas }\end{array}$} & $\begin{array}{l}\text { "...que decía que todo los casos de embarazadas específicamente, de menos de } 14 \text { años } \\
\text { había que denunciarla, no me acuerdo si eran todas las chicas que tenían actividad sexual, } \\
\text { pero era específico para las embarazadas de menos de } 14 \text { años" }\end{array}$ \\
\hline & $\begin{array}{l}\text { "...el San José la devuelve, independiente de la edad del pololo, si no se hace la denuncia } \\
\text { ellos no la atienden, entonces la chica vino, dijo, es que al hospital fui a la urgencia porque } \\
\text { había tenido alguna molestia y me dijeron que si ustedes no hacían la denuncia no me van } \\
\text { a atender el parto, entonces vengo para que me hagan la denuncia..." }\end{array}$ \\
\hline & $\begin{array}{l}\text { "Hasta el minuto, nosotros tenemos el lineamiento de la ley, que si tiene } 14 \text { años y el pololo } \\
\text { tiene } 15 \text { no vamos a hacer denuncia, si tiene } 13 \text { y el pololo tiene } 14 \text { no vamos a hacer } \\
\text { denuncia, pero no se qué vaya a pasar en el San José cuando ellos la reciban, si la van a } \\
\text { devolver para que hagamos la denuncia o ellos van a hacer la denuncia allá" }\end{array}$ \\
\hline & $\begin{array}{l}\text { "O sea, cuando hay una diferencia de } 2 \text { años o más, nosotros hacemos la denuncia que } \\
\text { corresponde, que generalmente nos llegan chicas de, no sé, } 13 \text { años con pololos de } 18 \\
\text { por ejemplo". }\end{array}$ \\
\hline \multirow[t]{2}{*}{ Quiebre confidencialidad } & $\begin{array}{l}\text { "La atención es } 100 \% \text { confidencial, cuando ellos llegan yo les enmarco la confidencialidad, } \\
\text { les digo que si ellos me dicen que si se van a hacer daño o van a hacer daño a un tercero } \\
\text { yo rompo la confidencialidad. En el caso de menores de } 14 \text { años... se rompe la confiden- } \\
\text { cialidad". }\end{array}$ \\
\hline & $\begin{array}{l}\text { "... bueno las menores de } 14 \text { años se le piden que vengan con un tutor mayor de edad. } \\
\text { Pero a las mayores de } 14 \text { años si ellas quieren venir solas o con la mamá, como que no se le } \\
\text { ponen muchos problemas... porque para manejarlo dentro del marco legal, de las menores } \\
\text { de } 14 \text { tienen que venir acompañadas...". }\end{array}$ \\
\hline \multirow[t]{5}{*}{ Indicación de anticoncepción } & $\begin{array}{l}\text {... vienen para acá, consultan y yo les entrego el anticonceptivo de forma inmediata, pero } \\
\text { también hay una evaluación del adolescente..." }\end{array}$ \\
\hline & $\begin{array}{l}\text { "... que tienen que evitar, tener relaciones sin protección [adolescentes menores de } 14 \\
\text { años] y si no nosotros no podemos entregarle el método, se les da la orientación de ir a } \\
\text { CEMERA". }\end{array}$ \\
\hline & $\begin{array}{l}\text { "...conversamos con ellas [adolescentes menores de } 14 \text { años], tratamos de buscar siempre } \\
\text { el adulto responsable. Lo que sí, cuando ya inicia actividad sexual o manifiesta el interés, } \\
\text { nunca la dejamos sin anticoncepción ..." }\end{array}$ \\
\hline & $\begin{array}{l}\text { "...si es muy chica, que nos ha pasado con } 12 \text { o } 13 \text { años, nos vienen a preguntar a no- } \\
\text { sotras, somos nosotras las que salimos a hablar con ellas... Que por la edad, debe venir } \\
\text { acompañada... Menores de 14, debe venir acompañada" }\end{array}$ \\
\hline & $\begin{array}{l}\text { "...un menor de } 14 \text { años viene a pedir anticonceptivo ahí nosotros no lo usamos, no lo } \\
\text { damos,... ahora si viene una niñita de } 13 \text { años yo le hago consejería, le digo todo y apenas } \\
\text { cumpla los } 14 \text { años, le voy a poner el implante o lo que sea" }\end{array}$ \\
\hline
\end{tabular}

Se identificaron variaciones en el proceso de denuncia a lo largo del tiempo. Varias matronas/ es relataban haber tenido malas experiencias en las primeras denuncias. Es a partir de éstas que se produjo un cambio en el proceder, en cuanto a no denunciar la actividad sexual cuando es consentida y no abusiva, pero manteniendo al mismo tiempo cierto resguardo profesional ante el temor de alguna sanción de tipo legal.

Fue generalizada la idea que debieran ser los equipos de salud, debidamente capacitados, quie- nes evalúen los casos de la menores de 14 años con actividad sexual y denunciar sólo cuando exista sospecha de agresión sexual.

\section{Discusión}

Se identificó un conocimiento parcial y ambiguo de los aspectos normativos y legales relativos a la atención de adolescentes para la prevención del embarazo. Sin embargo, existe un conocimiento 
generalizado de la normativa legal relativa al abuso sexual, la cual determina la diferenciación en la atención clínica de adolescentes mujeres menores de 14 años. Lo que de alguna manera muestra que, más allá de las normativas, existe la visión que son las mujeres quienes son víctimas de abuso, minimizando esta posibilidad en los varones. Una limitación del estudio fue no profundizar en las diferencias de género.

El abordaje clínico para adolescentes mayores es similar al de las adultas, viéndose expuestas a las barreras de acceso conocidas, como no contar con espacio diferenciado para la atención ${ }^{2}$. Es así que las adolescentes menores de 14 años, además de encontrarse con las barreras conocidas, se agregan los aspectos médicos legales ${ }^{2}$. En este sentido, es la Ley 19.927 la que determina en mayor medida el accionar de los profesionales en la atención de estas menores, al no diferenciar el abuso sexual de aquellos casos donde hay inicio temprano de la actividad sexual consentida y no abusiva. Es la interpretación de los proveedores de salud y las acciones que se derivan de ésta, lo que se instaura como una barrera de acceso a MAC y AE de las adolescentes menores de 14 años. Existe variabilidad en el proceder cuando estas adolescentes solicitan MAC o AE, las que se observan entre los profesionales de los distintos centros de atención, como dentro de un mismo centro. Por lo tanto, la resolución de la necesidad de atención de estas adolescentes queda condicionada al criterio e interpretación del ML del profesional que las atienda, en la medida que los profesionales no cuentan con orientaciones técnicas para el manejo de estos casos, es difícil promover el respeto por los derechos de los/las adolescentes a recibir una atención digna, de calidad, respetando los principios bioéticos $^{16}$. La Ley 20.418 establece que luego de la entrega de AE se informará a un adulto, no explicitando tener que actuar de igual forma en el caso de indicar un $\mathrm{MAC}^{17}$. Sin embargo, este artículo se interpreta en conjunto con la ley de abuso sexual, lo que condiciona la entrega de MAC a la presencia de un adulto, en el caso de menores de 14 años. El derecho a la confidencialidad se entiende para mayores de 14 años. De esta manera, el ML y en particular las leyes 19.927 y 20.418 , se trasforman en factores claves que enmarcan la diferenciación del abordaje clínico y la vivencia de inseguridad e incertidumbre en la atención para los proveedores, aspectos que se transforman en una barrera para una atención de calidad, que garantice el acceso oportuno, consejería y confidencialidad en la atención para la prevención del embarazo en adolescentes menores de 14 años.

Es así, que este puzzle legal lleva a los profesionales a considerar la actividad sexual siempre como una situación de abuso, en menores de 14 años, imposibilitando a los equipos de salud a aplicar criterios, discriminando entre los casos reales de abuso sexual de aquellos en que existe actividad sexual consentida y no abusiva. Este actuar obliga a judicializar la atención clínica, impidiendo que sea abordada integralmente. Sin desconocer que la actividad sexual a temprana edad pudiera estar asociada a problemáticas psicosociales, necesarias de identificar e intervenir ${ }^{18,19}$.

Está publicado que las adolescentes menores de 15 años tienen mayor riesgo biológico y psicosocial ante un embarazo y que el inicio sexual temprano se asocia a factores de vulnerabilidad ${ }^{20,21}$. Resulta preocupante que sea precisamente este grupo de mayor riesgo el que tiene más dificultades de acceso a la atención, quedando en más desprotección. Se sabe que a menor edad, aumenta la probabilidad que el embarazo de una adolescente sea producto de un abuso sexual, por lo cual es un antecedente que se debe indagar y que debe ser abordado por los equipos de salud de una manera diferente de aquellos embarazos en adolescentes menores producto de la actividad sexual consentida, ya que los riesgos e implicancias en salud y legales son diferentes.

De acuerdo a datos nacionales, el inicio de actividad sexual a los 15 años o antes ha aumentado de $14 \%$ a $26 \%$ entre los años 2010 y $2012^{22,23}$. De lo cual, podría inferirse que no se está resolviendo esta incipiente necesidad de salud. Consideramos necesario una reflexión a nivel de sociedad, autoridades de salud, educación y otras entidades sociales que logren explicar y definir la tendencia actual sobre la edad de inicio de la actividad sexual y su abordaje.

Si bien la legislación busca resguardar la integridad del niño y joven en materia de abusos sexuales, vulnera otra área relativa a los derechos en salud sexual y reproductiva (DSSR) garantizados en la legislación, como el acceso a los MAC y la confidencialidad. Estos DSSR se han garantizado en convenciones y acuerdos internacionales suscritos por el Estado de Chile. El Estado debiera asegurar el cumplimiento de los DSSR de 
los adolescentes, respetando la confidencialidad y autonomía progresiva, generando protocolos que otorgue un contexto de seguridad y claridad en cuanto a cómo proceder en el caso de menores de 14 años que solicitan MAC.

\section{Referencias}

1. Dides C, Benavente C, Morán JM. Diagnóstico de la situación del embarazo en la adolescencia en Chile. Santiago: 2008. Ministerio de Salud, FLACSO-Chile, UNFPA; 2008. Programa Género y Equidad.

2. Sadler M, Obach A, Luengo X, Biggs M. Estudio barreras de acceso a los servicios de salud para la prevención del embarazo adolescente en Chile. Santiago: Ministerio de Salud de Chile; 2011.

3. Dides C, Benavente C, Sáez I. Seguimiento a la Ley 20.418: Prevención del embarazo adolescente, educación sexual y anticoncepción de emergencia. Santiago: Ministerio de Salud y UNFPA; 2011. Plan Regional Andino de Prevención del Embarazo Adolescente.

4. Burdiles P, Santander R. Situación actual del embarazo adolescente en Chile. Santiago: Minsal; 2013. Programa Nacional de Salud Integral Adolescentes y Jóvenes.

5. Depto. Epidemiología MINSAL. Objetivos Sanitarios para la década 2000-2010. Boletín de vigilancia en Salud Pública de Chile 2002; 5: 1-12.

6. Código Penal y el Código Procesal Penal en materia de delitos de pornografía infantil. Ley número 19.927, Biblioteca del Congreso Nacional, (14-01-2004). [Consultado el 22 de mayo de 2012], disponible en http:// www.leychile.cl/N?i=220055\&f=2004-01-14\&p=

7. Código de procedimiento penal y otros cuerpos legales en materias relativas al delito de violación. Ley 19.617. Biblioteca del Congreso Nacional, (12-07-1999). [Consultado el 22 de mayo de 2012], disponible en https:// www.leychile.cl/N?i=138814\&f=1999-09-17\&p=

8. Código penal, Ley 28.704 que Modifica artículos relativos a los delitos contra la libertad sexual y excluye a los sentenciados de los derechos de gracia, indulto y conmutación de la pena. Congreso de la República del Perú, (13-03-2006). [Consultado el 8 de junio de 2015], Disponible en http://www2.congreso.gob. pe/Sicr/TraDocEstProc/TraDoc_condoc_2001.nsf/ d99575da99ebfbe305256f2e006d1cf0/e164b0a268f4db600525745700688b4d/\$FILE/SPL12494050413.pdf

9. Código procesal penal Argentino, delitos contra la integridad sexual, Ley 25.087. Información legislativa y documental, (14-5-1999). [Consultado el 8 de junio de 2015], Disponible en http://www.infoleg.gov.ar/infolegInternet/anexos/15000-19999/16546/texact.htm
10. Ley 20.418. Fija Normas sobre Información, Orientación y Prestaciones en Materia de Regulación de la Fertilidad. Biblioteca del Congreso Nacional, (28-01-2010). [Consultado el 5 de mayo de 2012]. Disponible en :http:// www.leychile.cl/N?i=1010482\&f=2010-02-02\&p=

11. Naciones Unidas. Informe de la Conferencia Internacional sobre la Población y el Desarrollo. Nueva York: Naciones Unidas; 1995. A/CONF.171/13/Rev.1

12. Decreto 830, Promulga Convención Sobre Los Derechos Del Niño. Biblioteca del Congreso Nacional, (20-111989). [Consultado el 6 de junio de 2012], disponible en https://www.leychile.cl/N?i=15824\&f=1990-09-27\&p=

13. Romero P, Oyarzún P. Anticoncepción en adolescentes ¿Qué dicen las leyes? Impacto de las leyes 19.927 y 20.418 en la Atención en salud de la adolescente al consultar por anticoncepción. Revista Sogia 2011; 18 (1): 8-16.

14. Hasen F. Documento de sistematización Encuentros de adolescentes y salud, en salud los jóvenes eligen. Santiago: Minsal; 2011.

15. Strauss A, Corbin J, Editores. Bases de la investigación cualitativa: técnicas y procedimientos para desarrollar la teoría fundamentada. Editorial Universidad de Antioquia; 2002.

16. Montero A, González E, Martínez V, Leal I, Bórquez B. Aspectos bioéticos relacionados con la necesidad y oportunidad de denunciar la actividad sexual en adolescentes menores de 14 años en Chile. En: Bioética en la toma de decisiones, 2da edición, FELAIBE, Guanajuato, 2013; 45-55.

17. Castro R, Díaz S, Galán G, López C, Matamala M. Normas Nacionales sobre Regulación de la Fertilidad. Santiago: Ministerio de Salud; 2007.

18. Estatuto Administrativo, Ley 18.834. Biblioteca del Congreso Nacional, (23-09-1989).

19. Código Penal de la República de Chile. Edición oficial del Código Penal aprobada por Decreto, No 417, (22-012010).

20. WHO Guidelineson Preventing Early Pregnancy and Poor Reproductive Outcomes Among Adolescents in Developing Countries, 2011.

21. Molina R, González E, Molina T. Madres niñas-adolescentes de 14 años y menos. Un grave problema de salud pública no resuelto en Chile. Rev Med Chile 2007; 135: 79-86.

22. Alt C, Aravena A, Argote L, Barretto M, Donoso A, Ferreiro JM, et al. Sexta Encuesta Nacional de Juventud. Santiago: Instituto Nacional de la Juventud; 2010.

23. Aubry M, Barreto M, Acevedo J, Foster B, Lobos F. Séptima Encuesta Nacional de Juventud. Santiago: Instituto Nacional de la Juventud; 2012. 